\title{
Preliminary Study: Immediate Effect of the Slow Deep Breathing Exercise and Sustained Maximal Inspiration with Volume-Oriented Incentive Spirometry on the Diffusing Lung Capacity in Healthy Young Participants
}

\author{
Araya Yankai ${ }^{1}$, Jirakrit Leelarungrayub ${ }^{1, *}$, \\ Rungtiwa Kanthain ${ }^{1}$ and James J Laskin ${ }^{2}$ \\ ${ }^{I}$ Department of Physical Therapy, Faculty of Associated Medical Sciences, Chiang Mai University, \\ Chiang Mai 50200, Thailand \\ ${ }^{2}$ School of Physical Therapy \& Rehabilitation Sciences, The University of Montana, \\ Missoula, MT 59812, United States
}

('Corresponding author's e-mail: donrawee.leela@cmu.ac.th)

Received: 12 June 2020, Revised: 1 August 2021, Accepted: 12 August 2021

\begin{abstract}
Slow-deep breathing exercise (SDBE) while using a volume-oriented type of incentive spirometry (VIS) device (SDBE/VIS) is one of the techniques in chest physical therapy designed to improve lung volume and oxygenation. However, the immediate effect of SDBE/VIS paired with a sustained maximal inspiration (SMI) on diffusing lung capacity (DLC), has not been documented. This preliminary study aimed to evaluate the immediate effect of SDBE/VIS paired with the SMI technique on DLC in healthy participants. Twenty healthy sedentary adults (11 males and 9 females) aged 20 to 23 years were recruited into this cross-over study and randomly assigned to one of 2 ordered groups; SDBE/VIS paired with SMI followed by SDBE/VIS alone or SDBE/VIS alone followed by SDBE/VIS with SMI. During each of the 2-testing sessions, the participants performed 3 sets of 5 SDBE/VIS trials which were controlled by ensuring that each participant reached their maximal vital capacity; derived from their pulmonary function test. The DLC for carbon monoxide (DLCO), alveolar volume (VA), and total lung capacity (TLC) was assessed prior to (baseline) and after each of the 5 experimental trials. A 3-min rest period was given between each set of 5 trials and 1 week between testing sessions.

The results demonstrated that SDBE/VIS paired with the SMI technique significantly increased both the DLCO ( $\mathrm{mL} / \mathrm{min} / \mathrm{mmHg}$ and \%) and VA (L and \%) when compared to the baseline values. Whereas, SDBE/VIS without using the SMI technique did not alter the DLCO, VA or the DLCO/VA. However, both SDBE/VIS with and without the SMI technique showed a significant increase the TLC (L and \%), with the TLC from SDBE/VIS paired with SMI was significantly higher than when the SDBE /VIS was performed without using the SMI technique. Thus, it appears that performing SDBE/VIS exercised paired with the SMI technique has a significant and positive effect on DLC.
\end{abstract}

Keywords: Diffuse lung capacity, Incentive spirometer, Slow deep breathing exercise, Sustained maximal inspiration

\section{Introduction}

Slow deep breathing exercise (SDBE) is one of many techniques used in physical therapy to address pulmonary system dysfunctions such as shortness of breath, abnormal breathing patterns, ineffective lung ventilation and gas exchange, as well as the pulmonary aspects of exercise intolerance [1]. The SDBE technique is applied clinically in several specific pulmonary conditions such as atelectasis, pleural effusion, and pneumothorax. Clinically the goal of SDBE is a reduction in the work of breathing and improved pulmonary ventilation [2]. SDBE can be performed with a focus on either costal or diaphragmatic breathing $[3,4]$ and is often combined with a sustained maximal inspiration (SMI) or holding of the breath at the end of maximal inspiration for $1-2 \mathrm{~s}$ [3]. The protocol of SDBE requires the patient to breathe in through the nose and out through the mouth, with the therapist facilitating the maneuver via costal or diaphragmatic/abdominal manual contacts [5]. A representative study with 
healthy volunteers showed that even an acute bout of deep breathing exercises significantly increased their vital capacity [6] and reduced both systemic and pulmonary arterial blood pressures [7]. Incentive spirometry (IS), a typical clinical tool to facilitate SDBE, is purported to help prevent atelectasis by improving chest wall flexibility and lung volumes. IS is used extensively as it is an activity that patients can perform effectively without the presence of a therapist [8]. The IS device was originally developed for patients undergoing abdominal surgery [9]. Nowadays, it is used widely in both acute and chronic care and comes in 2 forms: Flow-oriented incentive spirometry (FIS) and volume-oriented incentive spirometry (VIS), which are designed purportedly to address the respiratory muscles [10] and diaphragmatic function [11], respectively. The literature suggests that SDBE paired with a FIS along with the SMI technique could increase vital capacity (VC) [12]. While it appears that SDBE paired with a VIS (SDBE/VIS) has demonstrated significantly more effectiveness on lung volume, chest wall mobility [13], and diaphragm motion [12] than when SDBE is performed with a FIS. Moreover, the literature suggests that deep inspiratory breathing with a breath-hold or SMI is believed to allow more time for alveolar ventilation and oxygen uptake by the pulmonary circulation [14]. However, the potential beneficial effect on diffusing lung capacity (DLC) when SDBE/VIS is performed with the SMI technique has not been reported. Thus, this preliminary study was designed to evaluate the immediate effect of SDBE/VIS paired with the SMI technique on DLC in healthy participants.

\section{Materials and methods}

\section{Study design and recruitment of subjects}

This study was a prospective randomized cross-over study with the participants acting as their own controls, designed to examine the potential immediate effect between the SDBE/VIS with and without the SIM technique on DLC in young healthy participants. This study was approved by the ethics committee of the Faculty of Associated Medical Sciences, Chiang Mai University, Chiang Mai, Thailand (Study code: AMSEC-62EX-060), and conducted following the Declaration of Helsinki (2001). The sample size in this study was calculated using the data provided by Sivakumar et al. [6], who studied 12 healthy participants aged 20 - 40 years using SDBE. Forced vital capacity data measured before and after 2 min of SDBE assessed using a t-test was used to determine our sample size using $G^{*}$ Power (3.1.9.2) given an $\propto$-error prob $=0.05$, Power $(1-\beta$ error prob $)=0.85$, and effect size $=0.605$ with $\mathrm{t}$-test. Based on this calculation, a minimum of 18 healthy participants was required. We assumed a potential $20 \%$ sample loss therefore we recruited 20 participants. We used a sample of convenience and recruited participants from among the students who were studying at Chiang Mai University, Chiang Mai, Thailand. Data collection and evaluations were performed in an environmentally and temperature-controlled laboratory in the Department of Physical Therapy, Faculty of Associated Medical Sciences, Chiang Mai University, Chiang Mai, Thailand. Potential participants were required and screened to be: 1) nonsmokers (no history of smoking); 2) infrequent alcohol consumption; 3) free from any known pulmonary disease such as asthma and pneumonia; 4) no history of cardiac disease; 5) no chest wall or spinal deformity; 6) normal complete blood count; and 7) a normal pulmonary function test. Participants would be excluded if they did not meet all of the inclusion criteria if they reported consuming alcohol or exhaustive exercise prior to the testing session or any illness during the past week.

Twenty, sedentary (average body mass index was18.5 - $24.9 \mathrm{~kg} \cdot \mathrm{m}^{-2}$ and otherwise healthy participants aged 20 to 23 years of age volunteered to participate in this study. All participants were required to sign the consent form before being randomly assigned into one of the 2 experimental protocols orders: SBDE/VIS paired with SMI followed by SBDE/VIS alone or SBDE/VIS alone followed by SBDE/VIS paired with SMI. The 2 testing sessions were scheduled 1 week apart and the testing sessions were conducted by a chest physical therapist who had more than 10 years of experience in the clinic.

At each of the 2 testing sessions, the participants' pulmonary function was assessed via a standard pulmonary function test (PFT). They were when instructed on the proper procedures and use of the SBDE and VIS, ensuring that they were achieving their maximal vital capacity (determined by their PFT) with each practice trial. The baseline DLC test was completed within 2 min before the 3 sets of 5 experimental trials commenced and again within 2 min of the experimental trials being completed. A 3-min rest period was completed between each set of 5 trials.

\section{Pulmonary function test}

Pulmonary function test was performed on a portable spirometer (EasyOne Pro LAB, Medizintechnik AG, Tehnoparkstrasse, Zurich, Switzerland) using the normatively predicted lung 
volumes and capacities for the Thai population [15]. The PFT protocol was performed following the standard American Thoracic Society (ATS) guideline [16]. Specific lung function parameters of interest were forced vital capacity (FVC) (L and \%), forced expiratory volume in $1 \mathrm{~s}$ (FEV1) (L and \%), and FEV1/FVC ratio or the Tiffeneau-Pinelli index (absolute value and \%). All the PFT variables were determined from the best of the 3 trials as per the acceptability and reproducibility criteria outlined by the ATS.

\section{Slow-deep breathing exercise with volume-oriental incentive spirometer}

The VIS used in this study was the Voldyne ${ }^{\circledR} 5000$ (HUDSON RCI ${ }^{\circledR}$ USA), which had a target maximal volume set for an individual participant at the maximal vital capacity derived from their baseline PFT. When using the VIS, the participants were seated on a chair with their back supported and feet flat on the floor. When ready, the participants were instructed to complete 2 cycles of easy inhaling and exhaling by nasal breathing. At the end of the second easy cycle, they were instructed to perform a maximal expiration, insert the mouthpiece, and perform a maximal slow inspiration until the piston plate reached the volume target mark, this was repeated 5 times. When using the SMI technique, the participants were asked to hold their breath upon reaching their VIS volume target mark with sustained inflation for at least $3 \mathrm{~s}$. Whereas, when performing the SDBE/VIS protocol alone, the participants were asked not to hold and sustain their breath. They completed 3 sets of 5 trials of the prescribed technique during each of the 2 sessions. To prevent hyperventilation, a 3-min rest period was given between each set of 5 trials [17]. If signs or symptoms of confusion or sustained lightheadedness occurred, the session would be terminated following American Association for Respiratory Care (AARC) guidelines (2011) [18].

\section{Diffusing lung capacity test}

Classic single-breath DLC was determined using a commercial instrument (EasyOne Pro LAB, and Medizintechnik AG, Technoparkstrasse, Zurich, Switzerland). While seated on a chair with their back supported and feet flat on the floor, the participants were asked to quietly breathe room air via a mouthpiece while wearing a nose clip. They were then instructed to exhale to residual volume, were switched from room air to a gas mixture containing $0.3 \%$ carbon monoxide, $10 \%$ helium, $21 \%$ oxygen, and the balance of nitrogen, followed by a maximal inhalation and a breath-hold for $10 \mathrm{~s}$ before expiring slowly. The DLC test was conducted following the AARC Clinical Practice Guideline (1999) [19,20]. The maximal values of DLC for carbon monoxide (DLCO) (absolute value and \%), alveolar volume (VA) absolute value and \%), DLCO/VA ratio, and TLC (absolute value and \%) were recorded and analyzed from at least 2 acceptable tests with a 4-min rest interval between each DLC trial; a maximum of 4 attempts were allowed [21]. At each of the 2 testing sessions, DLC was assessed prior to the experimental trials and then again immediately after the trials were completed.

\section{Statistical analysis}

All data were analyzed statistically for normal distribution using the 1-sample KolmogorovSmirnov test and presented as mean with standard error of the mean (SEM) together with minimal and maximal values. The PFT data (FVC, FEV1 and FVC/FEV1 ratio) taken before the SDBE/VIS with and without the SMI technique were analyzed using paired t-tests. Whereas the parameters from DLC of 4 trials at before and after of both protocols were statistically analyzed using the 1-way ANOVA and Tukey post hoc method with SPSS software version 17.0 (SPSS Inc, Chicago IL, USA) for Windows. All of the tests were used with significance at a $p$-value of $<0.05$. Moreover, the effect size from the results with a Cohen value $(d)$, within and between the groups, was analyzed by G*Power 3.1.9.2, which presented 3 levels: Small $(d=0.2)$, moderate $(d=0.5)$, and large $(d>0.8)$, to confirm credible statistical analysis from the SPSS analysis [22].

\section{Results and discussion}

Twenty participants in this study consisted of 11 males and 9 females, aged $21.4 \pm 0.18$ years (20 to 23 years of age, with BMI of $21.90 \pm 0.32 \mathrm{~kg} \cdot \mathrm{m}^{-2}\left(18.32-23.88 \mathrm{~kg} \cdot \mathrm{m}^{-2}\right)$. The result of data distribution analysis showed a normal distribution of all parameters $(p>0.05)$. The baseline PFT test data for all 20 participants presented in Table 1 and were not significantly different $(p>0.05)$ after being statistically analyzed with a related paired t-test. 
Table 1 Pulmonary function parameters in the resting period before SDBE/VIS without and with the SMI technique in 20 participants.

\begin{tabular}{lcc}
\hline \multicolumn{1}{c}{ Parameters } & Before SDBE/VIS without SMI & Before SDBE/VIS with SMI \\
\hline FVC (L) & $3.59 \pm 0.13(2.39-4.42)$ & $3.62 \pm 0.14(2.44-4.48)$ \\
FVC (\%) & $94.91 \pm 2.49(80-112)$ & $94.35 \pm 2.40(81-111)$ \\
FEV1 (L) & $3.18 \pm 0.13(2.28-4.10)$ & $3.31 \pm 0.14(2.29-4.16)$ \\
FEV1 (\%) & $100.15 \pm 2.73(81-125)$ & $99.9 \pm 2.70(82-126)$ \\
FEV1/FVC & $0.92 \pm 0.01(0.82-0.99)$ & $0.91 \pm 0.01(0.80-0.99)$ \\
FEV1/FVC (\%) & $102.7 \pm 1.39(86-110)$ & $102.9 \pm 1.47(86-111)$ \\
\hline
\end{tabular}

Note: Data shows the mean \pm standard error of mean (SEM) $(\min -\max ) . \mathrm{FVC}=$ forced vital capacity, FEV1 = forced expiratory volume in $1 \mathrm{~s}$, SDBE =slow deep breathing exercise, VIS = volume-oriental type of incentive spirometry, $\mathrm{SMI}=$ sustained maximal inspiration, $\mathrm{L}=$ liter.

Table 2 Diffuse lung capacity before and after the SDBE/VIS without and with SMI technique in 20 participants.

\begin{tabular}{|c|c|c|c|c|c|c|c|}
\hline \multirow{2}{*}{ Parameters } & \multicolumn{2}{|c|}{ SDBE/VIS without SMI } & \multirow{2}{*}{$d$} & \multicolumn{2}{|c|}{ SDBE/VIS with SMI } & \multirow{2}{*}{$d$} & \multirow{2}{*}{$d^{\#}$} \\
\hline & Before & After & & Before & After & & \\
\hline $\begin{array}{c}\text { DLCO } \\
\left(\mathrm{mL} \cdot \mathrm{min}^{-1} \cdot \mathrm{mmHg}^{-1}\right)\end{array}$ & $\begin{array}{l}29.99 \pm 1.14 \\
(18.5-37.5)\end{array}$ & $\begin{array}{l}31.06 \pm 1.32 \\
(18.7-42.3)\end{array}$ & 0.52 & $\begin{array}{l}29.44 \pm 1.19 \\
(18.4-39.5)\end{array}$ & $\begin{array}{c}39.55 \pm 3.26^{*}, \# \\
(29-98)\end{array}$ & 0.71 & 0.59 \\
\hline DLCO (\%) & $\begin{array}{c}105.2 \pm 3.13 \\
(86-131)\end{array}$ & $\begin{array}{c}108.5 \pm 3.09 \\
(92-134)\end{array}$ & 0.56 & $\begin{array}{c}104.3 \pm 3.44 \\
(82-147)\end{array}$ & $\begin{array}{c}117.4 \pm 2.56^{*} \\
(98-142)\end{array}$ & 1.17 & 0.76 \\
\hline VA (L) & $\begin{array}{c}5.01 \pm 0.19 \\
(3.26-6.31)\end{array}$ & $\begin{array}{c}5.15 \pm 0.18 \\
(3.45-6.45)\end{array}$ & 0.91 & $\begin{array}{l}5.05 \pm 0.17 \\
(3.30-6.26)\end{array}$ & $\begin{array}{c}5.89 \pm 0.13^{*}, \# \\
(4.48-6.65)\end{array}$ & 1.36 & 0.91 \\
\hline VA (\%) & $\begin{array}{c}97.9 \pm 2.76 \\
(71-120)\end{array}$ & $\begin{array}{c}101.3 \pm 2.02 \\
(85-115)\end{array}$ & 0.34 & $\begin{array}{c}97.35 \pm 2.94 \\
(72-125)\end{array}$ & $\begin{array}{c}115.05 \pm 3.26^{*} \\
(96-144)\end{array}$ & 1.18 & 0.96 \\
\hline $\begin{array}{c}\mathrm{DLCO} / \mathrm{VA} \\
\left(\mathrm{ml} \cdot \mathrm{min}^{-1} \cdot \mathrm{mmHg}^{-1} \cdot \mathrm{l}^{-1}\right)\end{array}$ & $\begin{array}{c}5.7 \pm 0.31 \\
(4.19-7.35)\end{array}$ & $\begin{array}{c}5.93 \pm 0.16 \\
(4.82-7.64)\end{array}$ & 0.37 & $\begin{array}{c}5.84 \pm 0.17 \\
(4.31-7.14)\end{array}$ & $\begin{array}{c}6.09 \pm 0.22 \\
(4.42-7.86)\end{array}$ & 0.26 & 0.20 \\
\hline TLC (L) & $\begin{array}{c}4.65 \pm 0.15 \\
(3.41-6.36)\end{array}$ & $\begin{array}{l}5.97 \pm 0.21^{*} \\
(3.52-6.89)\end{array}$ & 1.0 & $\begin{array}{c}5.25 \pm 0.19 \\
(3.40-6.41)\end{array}$ & $\begin{array}{c}6.32 \pm 0.12^{*, \#} \\
(5.17-6.89)\end{array}$ & 0.69 & 0.51 \\
\hline TLC (\%) & $\begin{array}{l}97 \pm 1.67 \\
(73-121)\end{array}$ & $\begin{array}{c}119 \pm 2.13^{*} \\
(87-132)\end{array}$ & 1.8 & $\begin{array}{c}97.30 \pm 1.89 \\
(72-125)\end{array}$ & $\begin{array}{c}129.1 \pm 2.45^{*}, \# \\
(98-143)\end{array}$ & 1.49 & 0.82 \\
\hline
\end{tabular}

Note: Data shows the mean \pm standard error of the mean (min-max). DLCO $=$ diffuse capacity of the lungs for carbon monoxide, $\mathrm{VA}=$ alveolar volume, $\mathrm{TLC}=$ total lung capacity, and ${ }^{*} p<0.05$ compared to before the SDBE/VIS period within the group, ${ }^{\#} p<0.05$ compared to after the SDBE/VIS period from the Tukey post hoc method, $d$ =effect size, when compared between before and after the SDBE period within the group, $d^{\#}=$ effect size when compared at after session between the SDBE/VIS without and SDBE/VIS with SMI period.

The results from the DLC evaluation are shown in Table 2 and Figure 1. There was no significant difference in the baseline assessments between the 2 protocols. There were no significant pre to postsession changes in DLCO, VA, or DLCO/VA when SDBE/VIS was performed without the SMI technique. However, the TLC increased significantly with a large effect $(d>0.8)$ in terms of both absolute and percentage values.

When SDBE/VIS was performed paired with the SMI technique, there were significant improvements, with a large effect on DLCO (\%), VA (L and \%) and TLC $(\%)(d>0.8, \mathrm{p}<0.05)$; and moderate effect on DLCO ( $\mathrm{mL} / \mathrm{min} / \mathrm{mmHg})$ and TLC (L) $(0.5<d<0.8, p<0.05)$, when compared to their baseline measurements. Whereas, the SDBE/VIS alone showed significant increases only in TLC (L and $\%)$ with large effect $(d>0.8, p<0.05)$ when compared to baseline.

In addition, the data on DLCO ( $\mathrm{mL} / \mathrm{min} / \mathrm{mmHg})$, VA (L), and TLC (L \& \%) immediately after completion of SDBE/VIS with the SMI technique were also significantly different from SDBE/VIS without the SMI technique, with moderate to large effect, except for DLCO (\%), VA (\%), and DLCO/VA $\left(\mathrm{mL} \cdot \mathrm{min}^{-1} \cdot \mathrm{mmHg}^{-1} \cdot 1^{-1}\right)$. 

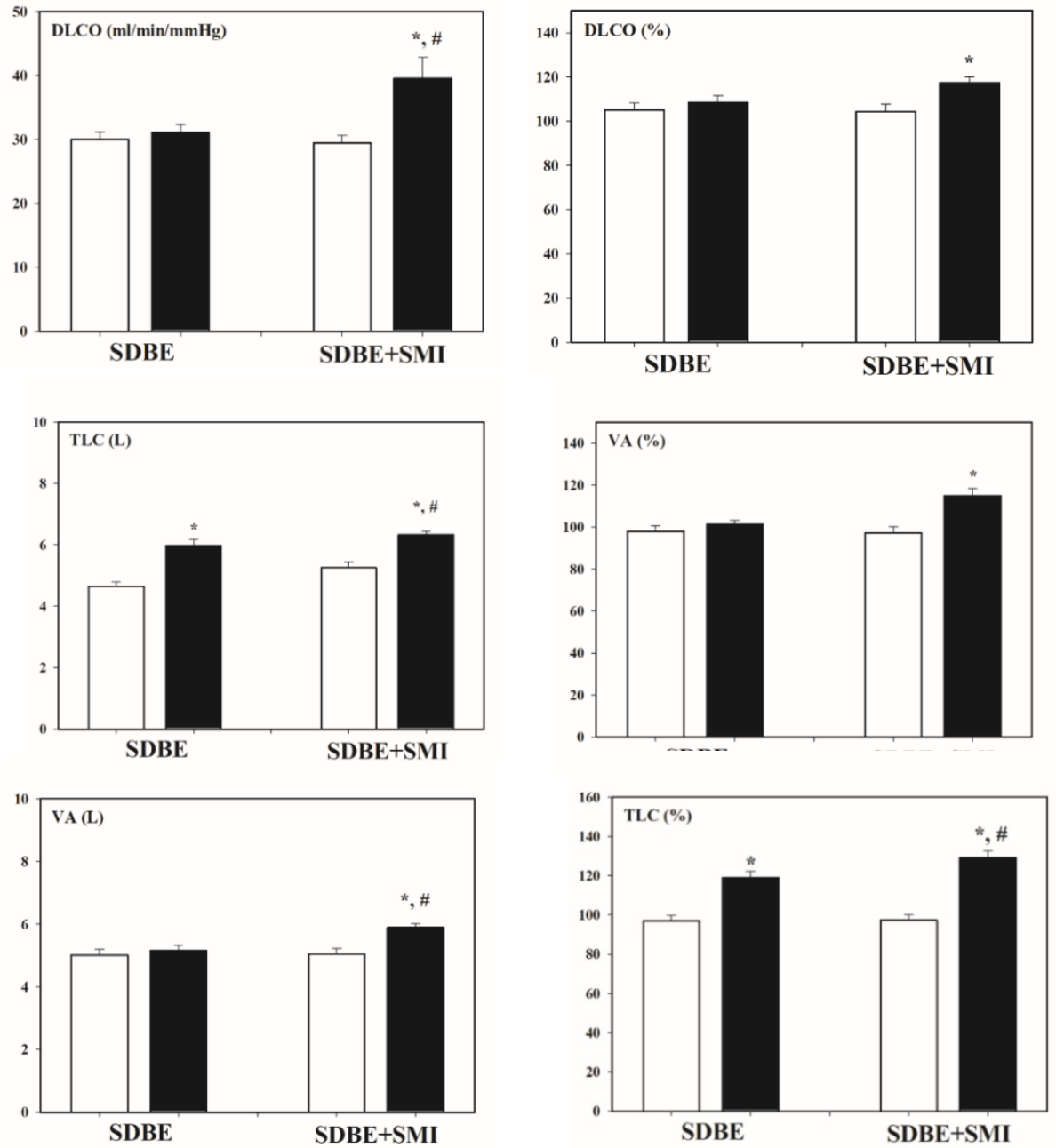

Figure 1 Diffuse lung capacity (DLC) parameters at before (white bar) and immediately after completely performed (back bar) the SDBE by VIS without and with SMI techniques DLCO = diffuse capacity of the lungs for carbon monoxide, $\mathrm{VA}=$ alveolar volume, $\mathrm{TLC}=$ total lung capacity. ${ }^{*} p<0.05$ when compared to before the SDBE/VIS period within the group, ${ }^{\#} p<0.05$ when compared to after the SDBE/VIS period.

\section{Discussion}

This preliminary study showed an immediate effect on DLC from performing SDBE using a VIS paired with the SMI technique. Although SDBE usually is applied manually with costal and diaphragmatic breathing exercises $[2,4,5]$, the Voldyne ${ }^{\circledR}$ volume-oriented IS has been recommended to be used by patients according to the AARC clinical practice guidelines [17]. IS has been claimed to be beneficial on the lung volume in various clinical populations such as Adults [23], the elderly [24], or patients with stroke [25]. However, there is evidence reported that IS alone did not help the overall recovery of lung function in patients who were developing pulmonary complications [26]. There is a gap in the literature looking specifically at the potential clinical benefits of adding the SMI technique to SDBE performed on a volume-oriented IS especially on VA and DLC. Thus, this preliminary study was designed and performed on healthy young adults where confounding factors to DLC have been eliminated and/or controlled [27]. Lung function can significantly impact DLC and affect DLCO determination, therefore it was important that the participants in this study presented with normal lung function. The participants in this study demonstrated lung function within normal limits (e.g. FVC $>70 \%$ of the predicted value) [28] and non-statistical differences of PFT before starting each session (e.g. FVC, TLC, and VA). If the VA is different between baseline or prior exercise, it will affect to DLCO result [29]; for instance, in those who are obese [30]. Therefore, to evaluate the effect of the SMI technique on DLCO, we repeated the PFT prior to each testing session. In addition, the precision of DLCO data has been 
demonstrated (absolute value and alveolar-volume corrected value) at baseline prior to the 2 testing sessions in 20 repeated measurements in 2 healthy individuals and showed good reliability with 2 and $3 \%$ (less than $5 \%$ ) [31].

A study looking at the immediate effect of SDBE/VIS showed that this technique did not alter the DLCO in healthy participants [7], which is consistent with our results of no significant differences in DLCO, VA, and DLCO/VA from SDBE /VIS alone. However, when the SMI technique was performed during SDBE/VIS, the results showed significantly increased DLCO (absolute value \& \%) and VA (absolute value \& \%), except the percentages of DLCO and VA when performing SDBE/VIS alone. These results support the suggestion that deep breathing with an end inspiratory-hold could promote collateral ventilation and redistribution of gas between lung segments [32,33].

The ratio of DLCO/VA between baseline and post SDBE/VIS with both experimental protocols was not statistically different, possibly because of the healthy condition of the participants with a close proportion of DLCO and VA. Whereas, TLC increased significantly in both SDBE/VIS with and without the SMI technique, and between baseline and after the experimental trials. However, the increase in TLC was significantly greater when SDBE/VIS was performed paired with SMI versus SDBE/VIS alone. The reasons for higher TLC from SDBE/VIS with SMI technique cannot be fully explained. However, it has been shown that TLC is significantly correlated with VA and the SMI technique appears to enhance VA [34].

There was a large effect $(d>0.8)$ of SDBE/VIS paired with SMI on DLCO (\%), VA (L and \%) and TLC (\%), and moderate effect $(d>0.5)$ on DLCO $\left(\mathrm{mL} \cdot \mathrm{min}^{-1} \cdot \mathrm{mmHg}^{-1}\right)$ and TLC (L). This large effect size was also calculated for TLC (L and \%)in SDBE/VIS alone. In addition, a moderate effect was demonstrated on DLCO (mL. $\left.\mathrm{min}^{-1} \cdot \mathrm{mmHg}^{-1}\right)$ and TLC (L), and a large effect on TLC (\%) and VA (L) were found with the SDBE/VIS with the SMI technique, when compared to SDBE/VIS alone. Finally, there was no statistical difference in DLCO (\%) with moderate effect and VA (\%) with large effect between the $2 \mathrm{SDBE} / \mathrm{VIS}$ conditions. It has been strongly suggested that a $p$-value may not be standardized in the case of a small sample size study, as calculation of Cohen $(d)$ or effect size of each result should be required. Cohen classifies the effect size as small $(d=0.2)$, moderate $(d=0.5)$, and large $(d>0.8)$ [22]. Thus, a significant result with a large effect point to be more robust. Notably, significant values of some parameters from SDBE/VIS with the SMI technique (Table 2) were consistent with its effect size results. Moreover, a non-significant result of DLCO/VA was presented in both experimental protocols, and the effect sizes were low, therefore, the results of DLCO/VA in a larger sample size should be performed [35]. An interesting result of this study is that SDBE/VIS alone appears to improve lung volume and TLC. An interesting study reports that SDBE/VIS could reduce the heart rate variability without altering the cardiac autonomic balance in healthy subjects aged 21 to 22 years [36] and SDBE/VIS protocol of taking 30 deep breaths hourly in the first 2 days post-cardiac surgery also improved oxygenation when applied with positive expiratory pressure [37]. Therefore, the clinical benefits of SDBE/VIS paired with the SMI technique in heart rate variability or other parameters in various patient populations such as post-cardiac surgery are just a few interesting areas to explore in the future besides populations with pulmonary dysfunction.

\section{Conclusions}

This study suggests that a specific slow deep breathing exercise, SDBE using the Voldyne ${ }^{\circledR}$ IS device can improve TLC, and that paired with SMI can show an immediate beneficial effect on DLCO and VA in healthy young adults.

\section{Limitations and suggestions}

Because this study was performed on 20 healthy subjects (11 males and 9 females), aged 20 to 23 years old, the effects of SMI during SDBE/VIS on DLC in other age groups and diseases cannot be generalized to clinical populations. However, breathing exercise has been suggested in an 8-week pulmonary rehabilitation program, which possibly showed benefits of DLCO in patients with chronic obstructive pulmonary disease (COPD) [38] or recruited in 2 weeks of training at least [6,12]. From the immediate effect on DLCO in this study, it can be concluded that the SMI technique should be added during SDBE/VIS as previously addressed report [39]. Nevertheless, specific SDBE/VIS with the SMI technique, especially the long-term effect has not been reported. Although the 20 participants in this study were justified and appropriate, continued work examining the acute and long-term effects with a larger sample size is still needed as well as in other age groups and different diseases, especially in patients who have impaired gas exchange at the alveolar basement membrane such as those with COPD [39]. 


\section{Acknowledgments}

The authors give thanks to the Prapatsorn Co., Ltd., Bangkok, Thailand, which supported the EasyOne Pro LAB spirometer for evaluating DLCO, and pulmonary function in this study.

\section{References}

[1] RL Claudia. Breathing exercise. In: CC Zadai (Ed.). Pulmonary management in physical therapy. $1^{\text {st }}$ ed. Churchill Livingstone, New York, 1992, p.135.

[2] A Hough. Physiotherapy in respiratory care. Nelson Thornes, Cheltenham, 2001.

[3] S Solomen and P Aaron. Breathing techniques - a review. Int. J. Phys. Educ. Sports Health 2015; 2, 237-41.

[4] J Bott, S Blumenthal, M Buxton, S Ellum, C Falconer, R Garrod, A Harvey, T Hughes, M Lincoln, C Mikelsons, C Potter, J Pryor, L Rimington, F Sinfield, C Thompson, P Vaughn and J White. Guidelines for the physiotherapy management of the adult, medical, spontaneously breathing patient. Thorax 2009; 64, i1-i51.

[5] DSR Vieira, LPS Mendes, NS Elmiro, M Velloso, RR Britto and VF Parreira. Breathing exercises: Influence on breathing patterns and thoracoabdominal motion in healthy subjects. Braz. J. Phys. Ther. 2014; 18, 544-52.

[6] G Sivakumar, K Prabhu, R Baliga, MK Pai and S Manjunatha. Acute effects of deep breathing for a short duration (2-10 minutes) on pulmonary functions in healthy young volunteers. Indian. J. Physiol. Pharmacol. 2011; 55, 154-9.

[7] G Bilo, M Revera, M Bussotti, D Bonacina, K Styczkiewicz, G Caldara, A Giglio, A Faini, A Giuliano, C Lombardi, K Kawecka-Jaszcz, G Manicia, P Agostoni and G Parati. Effects of slow deep breathing at high altitude on oxygen saturation, pulmonary and systemic hemodynamics. PlosOne 2012; 7, e49074.

[8] DL Frownfelter. Chest physical therapy. Core textbook of respiratory care practice. St.Louis, Mosby, 1994.

[9] WJ O'Donohue. Prevention and treatment of postoperative atelectasis: Can it and will it be adequately studied? Chest $1985 ; \mathbf{8 7}, 1-2$.

[10] P Agostini and S Singh. Incentive spirometry following thoracic surgery: What should we be doing? Physiotherapy 2009; 95, 76-82.

[11] AS Kumar, GK Alaparthi, AJ Augustine, ZC Pazhyaottayil, A Ramakrishna and SK Krishnakumar. Comparison of flow and volume incentive spirometry on pulmonary function and exercise tolerance in open abdominal surgery: A randomized clinical trial. J. Clin. Diagn. Res. 2016; 10, KC01-KC06.

[12] T Kotani, T Akazawa, T Sakuma, S Nagaya, M Sonoda, Y Tanaka, T Katogi, T Nemoto and S Minami. Effects of incentive spirometry on respiratory motion in healthy subjects using cine breathing magnetic resonance imaging. Ann. Rehabil. Med. 2015; 39, 360-5.

[13] DDM Paisani, AC Lunardi, CCBMD Silva, DC Porras, C Tanaka and CRF Carvalho. Volume rather than flow incentive spirometry is effective in improving chest wall expansion and abdominal displacement using optoelectronic plethysmography. Respir. Care 2013; 58, 1360-6.

[14] DJ Malone and J Adler. The patient with respiratory failure-preferred practice pattern $6 F$. In: S Irwin and JS Tecklin (Eds.). Cardiopulmonary physical therapy. $4^{\text {th }}$ ed. Mosby, Missouri, 2004, p. 372-99.

[15] W Dejsomritrutai, A Nana, KN Maranetra, B Chuaychoo, K Maneechotesuwan, P Wongsurakiat, N Chierakul, S Charoenratanakul, J Tscheikuna, W Juengprasert, T Suthamsmai and C Naruman. Reference spirometric values for healthy lifetime nonsmokers in Thailand. J. Med. Assoc. Thai. 2000; 83, 457-66.

[16] BH Culver, BL Graham, AL Coates, J Wanger, CE Berry, PK Clarke, TS Hallstrandm J Hankinson, DA Kaminsky, NR Maclntyre, MC McCormack, M Rosenfeld, S Stanojevic, DJ Weiner Recommendations for a standardized pulmonary function report. An official American thoracic society technical statement. Am. J. Respir. Crit. Care Med. 2017; 196, 1463-72.

[17] R Gosselink, K Schrever, P Cosps, H Witvrouwen, P De Leyn, T Troosters, A Lerut, G Deneffe and $\mathrm{M}$ Decramer. Incentive spirometry does not enhance recovery after thoracic surgery. Crit. Care Med. 2000; 28, 679-83.

[18] RD Restrepo, R Wettstein, L Wittnebel and M Tracy. Incentive spirometry: 2011. Respir. Care 2011; 56, 1600-4.

[19] AARC Clinical Practice Guideline. Single-breath carbon monoxide diffusing capacity. Respir. Care $1993 ; \mathbf{3 8}, 511-5$. 
[20] N Macintyre, RO Crapo, G Viegi, DC Johnson, CPMVD Grinten, V Brusasco, F Burgos, R Casaburi, A Coates, P Enright, P Gustafsson, J Hankinson, R Jensen, R McKay, MR Miller, D Navajas, OF Pedersen, R Pellegrino and J Wanger. Standardisation of the single-breath determination of carbon monoxide uptake in the lung. Eur. Respir. J. 2005; 26, 720-35.

[21] BL Graham, V Brusasco, F Burgos, BG Cooper, R Jensen, A Kendrick, NR Maclntyre, BR Thompson and J Wanger. 2017 ERS/ATS standards for single-breath carbon monoxide uptake in the lung. Eur. Respir. J. 2017; 49, 1600016.

[22] GM Sullivan and R Feinn. Using effect size - or why the p-value is not enough. J. Grad. Med. Educ. 2012; 4, 279-82.

[23] VF Parreira, GM Tomich, RR Britto and RF Sampaio.Assessment of tidal volume and thoracoabdominal motion using volume and flow-oriented incentive spirometers in healthy subjects. Braz. J. Med. Biol. Res. 2005; 38, 1105-12.

[24] AC Lunardi, DC Porras, RC Barbosa, DM Paisani, CCBMD Silva, C Tanaka and CRF Carvalho. Effect of volume-oriented versus flow-oriented incentive spirometry on chest wall volumes, inspiratory muscle activity, and thoracoabdominal synchrony in the elderly. Respir. Care 2014; 59, 420-6.

[25] IN Lima, GA Fregonezi, R Melo, EE Cabral, A Aliverti, TF Campos and GM Ferreira. Acute effects of volume-oriented incentive spirometry on chest wall volumes in patients after a stroke. Respir. Care 2014; 59, 1101-7.

[26] P Agostini, B Naidu, H Cieslik, R Steyn, PB Rajesh, E Bishay, MS Kalkat and S Singh. Effectiveness of incentive spirometry in patients following thoracotomy and lung resection including those at high risk for developing pulmonary complications. Thorax 2013; 68, 580-5.

[27] JO Park, IS Choi and KO Park. Normal predicted values of single-breath diffusing capacity of the lung in healthy nonsmoking adults. Kor. J. Intern. Med. 1986; 1, 178-84.

[28] DA Kaminsky, T Whitman and PW Callas. DLCO versus DLCO/VA as predictors of pulmonary gas exchange. Respir. Med. 2007; 101, 989-94.

[29] JM Hughes and NB Pride. In defense of the carbon monoxide transfer coefficient KCO (TL/VA). Eur. Respir. J. 2001; 17, 168-74.

[30] I Enache, M Oswald-Mammosser, S Scarfone, C Simon, JL Schlienger, B Geny and A Charloux. Impact of altered alveolar volume on the diffusing capacity of the lung for carbon monoxide in obesity. Respiration $2011 ; \mathbf{8 1}, 217-22$.

[31] N Kara-Junior. How to measure the precision of clinical study results. Rev. Bras. Oftalmol. 2014; 73, 133-4.

[32] J Mead, T Takishima and D Leith. Stress distribution in lungs: a model of pulmonary elasticity. $J$. Appl. Physiol. 1970; 28, 596-608.

[33] H Menkes and J Britt. Physical therapy rationale for physical therapy. Am. Rev. Respir. Dis. 1980; 122, 127-31.

[34] DA Kaminsky, A Daud and D Chapman. The relationship between the baseline alveolar volume-tototal lung capacity ratio and airway responsiveness. Respirology 2014; 19, 1046-51.

[35] CC Serdar, M Cihan, D Yucel and MA Serdar. Sample size, power and effect size revisited: simplified and practical approaches in pre-clinical, clinical and laboratory studies. Biochem. Med. (Zagreb). 2021; 31, 010502.

[36] E Tharion, P Samuel, R Rajalakshmi, G Gnanasenthi and RK Subramanian. Influence of deep breathing exercise on spontaneous respiratory rate and heart rate variability: A randomized controlled trial in healthy subjects. Indian J. Physiol. Pharmacol. 2012; 56, 80-7.

[37] C Urell, M Emtner, H Hedenstrom, A Tenling, M Breidenskog and E Westerdahl. Deep breathing exercises with positive expiratory pressure at a higher rate improve oxygenation in the early period after cardiac surgery - a randomised controlled trial. Eur. J. Cardiothorac. Surg. 2011; 40, 162-7.

[38] AEM Eltorai, TJ Martin, AS Eltorai, GL Baird, TT Healey and AH Daniels. Utility of inspiratory volume in incentive spirometry. R. I. Med. J. 2018; 10, 37-40.

[39] M Behnia, C Wheatley, A Avolio and B Johnson. Influence of resting lung diffuse on exercise capacity in patients with COPD. BMC. Pulm. Med. 2017; 17, 117. 